\title{
NEW TYPE I ANCIENT COMPACT SOLUTIONS OF THE YAMABE FLOW
}

\author{
PANAGIOTA DASKALOPOULOS, MANUEL DEL PINO, JOHN KING, AND NATASA SESUM
}

\begin{abstract}
We construct new ancient compact solutions to the Yamabe flow. Our solutions are rotationally symmetric and converge, as $t \rightarrow-\infty$, to two self-similar complete non-compact solutions to the Yamabe flow moving in opposite directions. They are type I ancient solutions.
\end{abstract}

\section{INTRODUCTION}

Let $\left(M, g_{0}\right)$ be a compact manifold without boundary of dimension $n \geq 3$. If $g=\bar{\varphi}^{\frac{4}{n-2}} g_{0}$ is a metric conformal to $g_{0}$, the scalar curvature $R$ of $g$ is given in terms of the scalar curvature $R_{0}$ of $g_{0}$ by

$$
R=\bar{\varphi}^{-\frac{n+2}{n-2}}\left(-\bar{c}_{n} \Delta_{g_{0}} \bar{\varphi}+R_{0} \bar{\varphi}\right)
$$

where $\Delta_{g_{0}}$ denotes the Laplace Beltrami operator with respect to $g_{0}$ and $\bar{c}_{N}=4(n-1) /(n-2)$.

In 1989 R. Hamilton introduced the Yamabe flow

$$
\frac{\partial g}{\partial t}=-R g
$$

as an approach to give yet another proof of the Yamabe problem on manifolds of positive conformal Yamabe invariant, using the flow and the parabolic techniques. The Yamabe flow (1.1) is the negative $L^{2}$-gradient flow of the total scalar curvature, restricted to a given conformal class. This was shown by S. Brendle $[1,2]$ (up to a technical condition in $\operatorname{dim} n \geq 6$ ). Significant earlier works in this directions include those by R. Hamilton [13], B. Chow [5], R. Ye [21], H. Schwetlick and M. Struwe [18] among many others. The Yamabe conjecture, was previously shown by R. Shoen via elliptic methods in his seminal work [17].

In the special case where the background manifold $M_{0}$ is the sphere $S^{n}$ and $g_{0}$ is the standard spherical metric $g_{S^{n}}$, the Yamabe flow evolving a metric $g=\bar{\varphi}^{\frac{4}{n-2}}(\cdot, t) g_{S^{n}}$ takes (after rescaling in time by a constant) the form of the fast diffusion equation

$$
\left(\bar{\varphi}^{\frac{n+2}{n-2}}\right)_{t}=\Delta_{S^{n}} \bar{\varphi}-c_{n} \bar{\varphi}, \quad c_{n}=\frac{n(n-2)}{4} .
$$

Starting with any smooth metric $g_{0}$ on $S^{n}$, it follows by the results in [5], [21] and [11] that the solution of (1.2) with initial data $g_{0}$ will become singular at some finite time $t<T$ and $g$ becomes spherical at time $T$, which means that after a normalization, the normalized flow converges to the spherical metric. In addition, $\bar{\varphi}$ becomes extinct at $T$.

A metric $g=\bar{\varphi}^{\frac{4}{n-2}} g_{S^{n}}$ may also be expressed as a metric on $\mathbb{R}^{n}$ via stereographic projection. It follows that if $g=\hat{\varphi}^{\frac{4}{n-2}}(\cdot, t) g_{\mathbb{R}^{n}}$ (where $g_{\mathbb{R}^{n}}$ denotes the standard metric on $\mathbb{R}^{n}$ ) evolves by the Yamabe flow (1.1), then $\hat{\varphi}$ satisfies (up to rescaling in time by a constant) the fast diffusion equation on $\mathbb{R}^{n}$

$$
\left(\hat{\varphi}^{p}\right)_{t}=\Delta \hat{\varphi}, \quad p:=\frac{n+2}{n-2} .
$$


Observe that if $g=\hat{\varphi}^{\frac{4}{n-2}}(\cdot, t) g_{\mathbb{R}^{n}}$ represents a smooth solution when lifted to $S^{n}$, then $\hat{\varphi}(\cdot, t)$ satisfies the growth condition

$$
\hat{\varphi}(z, t)=O\left(|z|^{-(n-2)}\right), \quad \text { as }|z| \rightarrow \infty .
$$

Definition 1.1 (Type I and type II ancient solutions). The solution $g=\bar{\varphi}(\cdot, t)^{\frac{4}{n-2}} g_{0}$ to (1.1) is called ancient if it exists for all time $t \in(-\infty, T)$, where $T<\infty$. We will say that the ancient solution $g$ is type $I$, if its Riemannian curvature satisfies

$$
\limsup _{t \rightarrow-\infty}\left(|t| \max _{M_{0}}|\operatorname{Rm}|(\cdot, t)\right)<\infty .
$$

An ancient solution which is not type I, will be called type II.

The simplest example of an ancient solution to the Yamabe flow on $S^{n}$ is the family of contracting spheres. They are special solutions $\bar{\varphi}$ of (1.2) which depend only on time $t$ and satisfy the ODE

$$
\frac{d \bar{\varphi}^{\frac{n+2}{n-2}}}{d t}=-c_{n} \bar{\varphi}
$$

They are given by

$$
\bar{\varphi}_{S}(p, t)=\left(\frac{4}{n-2} c_{n}(T-t)\right)^{\frac{n-2}{4}}, \quad p \in S^{n}
$$

and represent a sequence of round spheres shrinking to a point at time $t=T$. They are shrinking solitons and type I ancient solutions.

King solutions: They were discovered by J.R. King [14]. They can be expressed on $\mathbb{R}^{n}$ in closed from (after stereographic projection), namely $g=\hat{\varphi}_{K}(\cdot, t)^{\frac{4}{n-2}} g_{\mathbb{R}^{n}}$, where $\hat{\varphi}_{K}$ is the radial function

$$
\hat{\varphi}_{K}(z, t)=\left(\frac{a(t)}{1+2 b(t)|z|^{2}+|z|^{4}}\right)^{\frac{n-2}{4}}, \quad z \in \mathbb{R}^{n}
$$

and the coefficients $a(t)$ and $b(t)$ satisfy a certain system of ODEs. The King solutions are not solitons and may be visualized, as $t \rightarrow-\infty$, as two Barenblatt self-similar solutions "glued" together to form a compact solution to the Yamabe flow. They are type I ancient solutions.

Let us make the analogy with the Ricci flow on $S^{2}$. The two explicit compact ancient solutions to the two dimensional Ricci flow are the contracting spheres and the King-Rosenau solution [14], [15], [16]. The latter one is the analogue of the King solution (1.5) of the Yamabe flow. The difference is that the King-Rosenau Ricci flow ancient solution is type II, while the King Yamabe flow solution is type I.

It has been showed by Daskalopoulos, Hamilton and Sesum [8] that the spheres and the King-Rosenau solution are the only compact ancient solutions to the two dimensional Ricci flow. The natural question to raise is whether the analogous statement holds true for the Yamabe flow, that is, whether the contracting spheres and the King solution are the only compact ancient solutions to the Yamabe flow. This occurs not to be the case as the following discussion shows.

Indeed, in [7] the existence of a new class of type II ancient radially symmetric solutions of the Yamabe flow (1.2) on $S^{n}$ was shown. These new solutions, as $t \rightarrow-\infty$, may be visualized as two spheres joined by a short neck. Their curvature operator changes sign. We will refer to them as towers of moving bubbles.

Since the towers of moving bubbles are shown to be type II ancient solutions, while the contracting spheres and the King solutions are of type I, one may still ask whether the latter two are the only ancient 
compact type I solutions of the Yamabe flow on $S^{n}$, equation (1.2). In this work we will observe that this is not the case, as will show the existence of other ancient compact type I solutions on $S^{n}$.

It is simpler to work in cylindrical coordinates, so let us first describe the coordinate change. Let $g=\hat{\varphi}^{\frac{4}{n-2}}(\cdot, t) g_{\mathbb{R}^{n}}$ be a radially symmetric solution of (1.3). For any $T>0$ the cylindrical change of variables is given by

$$
\varphi(x, \tau)=(T-t)^{-\frac{1}{p-1}} r^{\frac{2}{p-1}} \hat{\varphi}(y, t), \quad x=\ln |y|, \tau=-\ln (T-t) .
$$

In this language equation (1.3) becomes

$$
\left(\varphi^{p}\right)_{\tau}=\varphi_{x x}+\alpha^{-1} \varphi^{p}-\beta \varphi, \quad \beta=\frac{(n-2)^{2}}{4}, \quad \alpha=\frac{p-1}{p}=\frac{n+2}{4} .
$$

By suitable scaling we can make the two constants $\alpha$ and $\beta$ in (1.7) equal to 1 , so that from now on we will consider the equation

$$
\left(\varphi^{p}\right)_{\tau}=\varphi_{x x}+\varphi^{p}-\varphi
$$

It is well known (c.f. in [20], Section 3.2.2 and [10,9]) that for any given $\lambda \geq 0$ equation (1.8) admits an one parameter family of traveling wave solutions of the form

$$
\phi_{\lambda}(x, \tau)=\psi_{\lambda}(x-\lambda \tau), \quad y:=x-\lambda \tau
$$

which admit the behavior

$$
\psi_{\lambda}(y)=O\left(e^{y}\right), \quad \text { as } y \rightarrow-\infty .
$$

It follows that $\psi:=\psi_{\lambda}$ satisfies the equation

$$
\psi_{y y}+\lambda\left(\psi^{p}\right)_{y}+\psi^{p}-\psi=0
$$

and they are unique up to translations of the self-similar variable $y$, given the (1.9). The solutions $\varphi_{\lambda}$ define Yamabe shrinking solitons which correspond to smooth self-similar solutions of (1.3) when expressed as metrics on $\mathbb{R}^{n}$ (the smoothness follows from condition (1.9)). It was shown in [10] that they are type I ancient solutions. Solutions of (1.10) with $\lambda=0$ correspond to the steady states of equation (1.8) and they represent geometrically the standard metric on the sphere.

When $\lambda>0$, solutions to (1.10) with behavior (1.9) define smooth complete and non-compact Yamabe solitons (shrinkers) which all have cylindrical behavior at infinity, namely

$$
\psi_{\lambda}(y)=1+o(1), \quad \text { as } y \rightarrow+\infty .
$$

In [9] the asymptotic behavior, up to second order, of these solutions was shown. Let us next describe this behavior for the case $\lambda \geq 1$ which will be heavily used in this work. For values of $\lambda$ in the range $0<\lambda<1$, the behavior of the solutions $v_{\lambda}$ was also studied in [9] and it is more complex as it differs for dimensions $3 \leq N \leq 6$ and $N \geq 6$.

When $\lambda \geq 1$, it is shown in Theorem 1.1 in [9], that there exists a unique solution $\psi_{\lambda}$ of (1.10) which is monotone increasing, satisfies

and has the asymptotic behavior

$$
\psi_{\lambda}(0)=\frac{1}{2}
$$

$$
\psi_{\lambda}(y)=O\left(e^{y}\right), \quad \text { as } y \rightarrow-\infty \quad \text { and } \quad \psi_{\lambda}(y)=1-C_{\lambda} e^{-\gamma_{\lambda} y}+o\left(e^{-\gamma_{\lambda} y}\right), \quad \text { as } y \rightarrow+\infty
$$

for some constants $\gamma_{\lambda}>0$ and $C_{\lambda}>0$ (depending on $\lambda$ ). The exponent $\gamma_{\lambda}$ satisfies the equation

$$
\gamma^{2}-\lambda p \gamma+(p-1)=0
$$


and for $\lambda>1$ is given by the smallest of the roots of this equation, that is

$$
\gamma_{\lambda}=\frac{\lambda p-\sqrt{\lambda^{2} p^{2}-4(p-1)}}{2}>0
$$

When $\lambda=1$, equation (1.10) admits the explicit Barenblatt solution

$$
\psi_{1}(y)=\left(\frac{1}{1+c_{p} e^{-(p-1) y}}\right)^{1 /(p-1)}, \quad p-1=\frac{1}{n-2}
$$

with $c_{p}$ chosen so that $\psi_{1}(0)=1 / 2$.

We will establish in this work the existence of a five parameter family of solutions $\varphi_{\lambda, \lambda^{\prime}, h, h^{\prime}, k}$ of (1.8) with $\left(\lambda, \lambda^{\prime}, h, h^{\prime}, k\right) \in \mathbb{R}^{5}$ and $\lambda, \lambda^{\prime}>1, k \geq 0$. Let us next summarize our construction. First, note that because of the nonlinearity of the time derivative in (1.8) it is more natural to define our solutions in terms of the pressure function

$$
u:=\varphi^{-\frac{4}{n-2}}=\varphi^{-(p-1)}
$$

which satisfies the equation

$$
p u_{\tau}=u u_{x x}-\frac{p}{p-1} u_{x}^{2}+(p-1)\left(u^{2}-u\right) .
$$

This becomes also apparent when one looks at the King solutions (1.5), which in terms of the pressure function $u:=\varphi_{K}^{-(p-1)}$ become polynomials in the radial variable $r=|z|$.

In terms of the pressure function, equation (1.16) admits an one parameter family (unique up to translations) of traveling wave solutions of the form $u_{\lambda}(x, \tau)=v_{\lambda}(x-\lambda \tau)$ with profile $v_{\lambda}=\psi_{\lambda}^{-(p-1)}$, with $\psi_{\lambda}$ the solution of (1.10) as described above. It follows that $v:=v_{\lambda}$ satisfies the equation

$$
v v_{y y}-\frac{p}{p-1} v_{y}^{2}+\lambda p v_{y}+(p-1)\left(v^{2}-v\right)=0 .
$$

Imposing the condition that

$$
v_{\lambda}(0)=2
$$

which is equivalent to $\psi_{\lambda}(0)=1 / 2$, it follows from (1.12) that each $v_{\lambda}$ satisfies the asymptotic behavior

$$
v_{\lambda}(y)=O\left(e^{-(p-1) y}\right), \quad \text { as } y \rightarrow-\infty \quad \text { and } \quad v_{\lambda}(y)=1+C_{\lambda} e^{-\gamma_{\lambda} y}+o\left(e^{-\gamma_{\lambda} y}\right), \quad \text { as } y \rightarrow+\infty
$$

with $\gamma_{\lambda}$ satisfying (1.13)-(3.10) and $C_{\lambda}$ a constant uniquely determined in terms of $\lambda$ and $p$. In addition each $v_{\lambda}$ is monotone decreasing in $y$, since it is known that $\psi_{\lambda}$ is monotone increasing. Notice also that in terms of the pressure function $\varphi_{1}:=\psi_{1}^{-(p-1)}$ the Barenblatt solution in (1.15) are given by

$$
v_{1}(y)=1+c_{p} e^{-(p-1) y} .
$$

We will next describe the building blocks in our construction of the five parameter family $u_{\lambda, \lambda^{\prime}, h, h^{\prime}, k}$ of ancient solutions of equation (1.16), which will be the main focus in this work. Assume that

$$
u_{\lambda, h}(x, \tau):=v_{\lambda}(x-\lambda \tau+h)
$$

is a traveling wave solution of (1.16) for a parameter $\lambda>1$ and $h \in \mathbb{R}$ and let $u_{\lambda^{\prime}, h^{\prime}}$ be another such solution for a different choice of parameters $\lambda^{\prime}>1$ and $h^{\prime} \in \mathbb{R}$. Since equation (1.16) is invariant under reflection $x \rightarrow-x$, it follows that

$$
\hat{u}_{\lambda^{\prime}, h^{\prime}}(x, \tau):=u_{\lambda^{\prime}, h^{\prime}}(-x, t)=v_{\lambda}\left(-x-\lambda^{\prime} \tau+h^{\prime}\right)
$$


is also a solution to (1.16). It corresponds to another traveling wave of (1.17) which travels in the opposite direction than $u_{\lambda^{\prime}, h^{\prime}}$. The solution $u_{\lambda, h}(\cdot, \tau)$ is monotone decreasing in $x$ while $\hat{u}_{\lambda^{\prime}, h^{\prime}}(\cdot, \tau)$ is monotone increasing. Moreover, it follows from (1.18) that $u_{\lambda, h}, \hat{u}_{\lambda^{\prime}, h^{\prime}}$ satisfy the asymptotics

$$
u_{\lambda, h}(x, \tau)=O\left(e^{-(p-1) x}\right), \text { as } x \rightarrow-\infty \quad \text { and } \quad \hat{u}_{\lambda^{\prime}, h^{\prime}}(x, \tau)=O\left(e^{x(p-1)}\right) \text { as } x \rightarrow+\infty
$$

and also

$$
u_{\lambda, h}(x, \tau)=1+C_{\lambda} e^{-\gamma_{\lambda}(x-\lambda \tau+h)}+o\left(e^{-\gamma_{\lambda}(x-\lambda \tau+h)}\right), \quad \text { as } x-\lambda \tau+h \rightarrow+\infty .
$$

and

$$
\hat{u}_{\lambda^{\prime}, h^{\prime}}(x, \tau)=1+C_{\lambda} e^{\gamma_{\lambda^{\prime}}\left(x+\lambda^{\prime} \tau-h\right)}+o\left(e^{\gamma_{\lambda^{\prime}}\left(x+\lambda^{\prime} \tau-h\right)}\right), \quad \text { as } x+\lambda^{\prime} \tau-h \rightarrow-\infty .
$$

with $\gamma_{\lambda}, \gamma_{\lambda^{\prime}}$ given by (3.10) and $C_{\lambda}>0, C_{\lambda^{\prime}}>0$ depending only on $\lambda$.

Equation (1.16) also admits an one parameter family of space independent ancient solutions $\xi_{k}:=\xi_{k}(\tau)$ which are the solutions of the ode

$$
\frac{d}{d \tau} \xi(\tau)=\frac{p-1}{p}\left(\xi^{2}(\tau)-\xi(\tau)\right)
$$

and correspond to the cylindrical solution of the Yamabe flow. Solving this equation gives

$$
\xi_{k}(\tau)=\frac{1}{1-k e^{\frac{p-1}{p} \tau}} .
$$

for a constant $k \in \mathbb{R}$. From now on will take $k \geq 0$ which implies that $\xi_{k}(\tau) \geq 1$ and $\xi_{k}(\tau)>1$, for $k>0$. Also, since $p>1$, we have

$$
\xi_{k}(\tau)=1+k e^{\frac{p-1}{p} \tau}+O\left(e^{\frac{2(p-1)}{p} \tau}\right), \quad \text { as } \tau \rightarrow-\infty .
$$

We will show the existence of five parameter class of ancient solutions $u_{\lambda, \lambda^{\prime}, h, h^{\prime}, k}$ of equation (1.16) with $\lambda, \lambda^{\prime}>1, k \geq 0$ and $h, h^{\prime} \in \mathbb{R}$, which as $\tau \rightarrow-\infty$ may be visualized as the two traveling wave solutions, $u_{\lambda, h}$ (traveling on the left) and $\hat{u}_{\lambda^{\prime}, h^{\prime}}$ (traveling on the right) and a cylinder $\xi_{k}(\tau)$ in the middle. In fact, we will show in section 4 that for $\tau<<0, u_{\lambda, \lambda^{\prime}, h, h^{\prime}, k}(\cdot, \tau)$ satisfies

$$
w_{\lambda, \lambda^{\prime}, h, h^{\prime}, k}^{-}(\cdot, \tau) \leq u_{\lambda, \lambda^{\prime}, h, h^{\prime}, k}(\cdot, \tau) \leq w_{\lambda, \lambda^{\prime}, h, h^{\prime}, k}^{+}(\cdot, \tau)
$$

with

$$
w_{\lambda, \lambda^{\prime}, h, h^{\prime}, k}^{-}:=\max \left(v_{\lambda}(x-\lambda \tau+h), \xi_{k}(\tau), v_{\lambda^{\prime}}\left(-x-\lambda^{\prime} \tau+h^{\prime}\right)\right)
$$

and

$$
w_{\lambda, \lambda^{\prime}, h, h^{\prime}, k}^{+}:=v_{\lambda}\left(x-\lambda \tau\left(1-q e^{\frac{p-1}{p} \tau}\right)+h\right)+v_{\lambda^{\prime}}\left(-x-\lambda^{\prime} \tau\left(1-q e^{\frac{p-1}{p} \tau}\right)+h^{\prime}\right)+\xi_{k}(\tau)-2
$$

with $q=q(p)>0$. It is clear that $w_{\lambda, \lambda^{\prime}, h, h^{\prime}, k}^{-}$is a subsolution of equation (1.16). The main observation in our construction is that $w_{\lambda, \lambda^{\prime}, h, h^{\prime}, k}^{+}$is a supersolution of equation (1.16) with the appropriate choice of the parameter $q$.

Equivalently $\varphi_{\lambda, \lambda^{\prime}, h, h^{\prime}, k}:=u_{\lambda, \lambda^{\prime}, h, h^{\prime}, k}^{-\frac{1}{p-1}}$ defines a solution of (1.8) and we recall that $p-1=\frac{4}{n-2}$. Let $g_{\lambda, \lambda^{\prime}, h, h^{\prime}, k}(\tau):=\varphi_{\lambda, \lambda^{\prime}, h, h^{\prime}, k}^{\frac{4}{n-2}}(\cdot, \tau) g_{c y l}$ denote the metric on the cylinder $\mathbb{R} \times S^{n-1}$ defined in terms of $\varphi_{\lambda, \lambda^{\prime}, h, h^{\prime}, k}$, where $g_{c y l}:=d x^{2}+g_{S^{n-1}}$ denotes the standard cylindrical metric. We have seen that (1.8) is equivalent to $g_{\lambda, \lambda^{\prime}, h, h^{\prime}, k}$ satisfying the rescaled Yamabe flow $g_{\tau}=-(R-1) g$. In addition we will show that $\varphi_{\lambda, \lambda^{\prime}, h, h^{\prime}, k}$ when lifted on $S^{n}$ defines a smooth ancient type I solution to the Yamabe flow on $S^{n} \times(-\infty, T)$. Our main result is summarized as follows. 
Theorem 1.1. For any $\left(\lambda, \lambda^{\prime}, h, h^{\prime}, k\right) \in \mathbb{R}^{5}$ such that $\lambda, \lambda^{\prime}>1, k \geq 0$, there exists an ancient solution $\varphi_{\lambda, \lambda^{\prime}, h, h^{\prime}, k}$ of (1.8) defined on $\mathbb{R} \times(-\infty, T)$, for some $T=T_{\lambda, \lambda^{\prime}, h, h^{\prime}, k} \in(-\infty,+\infty]$, which as $\tau \rightarrow-\infty$ may be visualized as the two traveling wave solutions, $u_{\lambda, h}$ (traveling on the left) and $\hat{u}_{\lambda^{\prime}, h^{\prime}}$ (traveling on the right) and a cylinder $\xi_{k}(\tau)$ in the middle. More precisely, it satisfies the bound

$$
\left(w_{\lambda, \lambda^{\prime}, h, h^{\prime}, k}^{-}\right)^{-\frac{n-2}{4}}(\cdot, \tau) \leq \varphi_{\lambda, \lambda^{\prime}, h, h^{\prime}, k}(\cdot, \tau) \leq\left(w_{\lambda, \lambda^{\prime}, h, h^{\prime}, k}^{+}\right)^{-\frac{n-2}{4}}(\cdot, \tau), \quad \text { for } \tau<<0 .
$$

The metric $g_{\lambda, \lambda^{\prime}, h, h^{\prime}, k}(\tau):=\varphi_{\lambda, \lambda^{\prime}, h, h^{\prime}, k}^{\frac{4}{n-2}}(\cdot, \tau) g_{c y l}$ when lifted on $S^{n}$ defines a smooth ancient solution of the rescaled Yamabe flow $g_{\tau}=-(R-1) g$, on $S^{n} \times(-\infty, T)$. This is a type I ancient solution in the sense that the norm of its curvature operator is uniformly bounded in time $-\infty<\tau \leq \tau_{0}$, for all $\tau_{0}<T$.

Remark 1.1. It follows from Theorem 1.1 that the corresponding solution to the unrescaled Yamabe flow (1.1) is a type I ancient solution in the sense of Definition 1.1.

Remark 1.2. [Similarities with the KPP equation and further discussion] Equation (1.8) resembles the well known semilinear KPP equation

$$
\varphi_{\tau}=\varphi_{x x}+f(\varphi)
$$

for a nonlinearity $f(\varphi)$ which satisfies certain growth assumptions including $f(\varphi)=\varphi^{p}-\varphi$ as in (1.8). It is well known that equation (1.31) possesses a family of traveling wave solutions $\psi_{\lambda}, \lambda \geq \lambda_{*}$ with similar behavior as those of equation (1.10) described above. F. Hamel and N. Nadirashvili showed in [12] the existence of ancient solutions $\varphi_{\lambda, \lambda^{\prime}, h, h^{\prime}, k}$ to (1.31) which are similar to those in Theorem 1.1. The method in [12] exploits the semilinear character of equation (1.31). The error of approximation is estimated in a rather precise manner by the corresponding solution of the linear equation $\zeta_{\tau}=\zeta_{x x}+f^{\prime}(0) \zeta$. This is done simply by using the heat kernel. The same method cannot be applied to the quasilinear equation (1.8), which also becomes singular as $x \rightarrow \pm \infty$. Despite the singular nature of our equation, we are still able to obtain similar precise bounds as in [12] by the construction of the appropriate super-sub solutions.

It would be interesting to explore whether similar methods, using barriers, can be used to provide the construction of ancient solutions from solitons (self-similar solutions) in other parabolic PDE and in particular in geometric flows.

In our previous work [6] we showed the existence of a four parameter family $g_{\lambda, \lambda^{\prime}, h, h^{\prime}}$ of type I ancient solutions of the Yamabe flow (1.1). These solutions correspond to the case $k=0$ in our five parameter family $g_{\lambda, \lambda^{\prime}, h, h^{\prime}, k}$ which is also covered by Theorem 1.1. The methods of the construction in [6], which are based on integral bounds and a'priori estimates, are different than in this work. Our super-sub solution method of this work allows us to obtain much sharper bounds on the solution as $\tau \rightarrow-\infty$. These bounds are similar to those for the KPP equation in [12].

The outline of the paper is as follows. In section 2 we will review the construction of the King solutions proven to exist in [14] and show their relevance to our construction. In section 3 we will show that $w_{\lambda, \lambda^{\prime}, h, h^{\prime}, k}^{+}$given by (1.30) defines a supersolution of equation (1.16) for an appropriate choice of parameters $q$ and $d$. This will constitute the main step in our proof. Section 4 will be devoted to the proof of the existence of the ancient solution $u_{\lambda, \lambda^{\prime}, h, h^{\prime}, k}$ as stated in Theorem 1.1. In the final section 5 we will show that our solution defines a type I ancient solution of the Yamabe flow. 


\section{The King SOlutions}

In this section we will review the existence and properties of the King solutions found in [14] and show the similarities with our construction of $u_{\lambda, \lambda^{\prime}, h, h^{\prime}, k}$ as stated in Theorem 1.1.

One looks for a radially symmetric solution $\hat{\varphi}_{K}(z, t)$ of equation (1.3) such that the pressure function $\hat{u}_{K}:=\hat{\varphi}_{K}^{-(p-1)}$ is a polynomial in $r=|z|$ with coefficients depending on time $t$. It turns out that the polynomial is of degree four, that is, $\hat{u}_{K}$ has the form

$$
\hat{u}_{K}(r, t):=a(t)^{-1}\left(1+2 b(t) r^{2}+r^{4}\right), \quad r=|z|, z \in \mathbb{R}^{n}
$$

where $a(t), b(t)$ are not defined explicitly but satisfy a system of ode's (c.f. in [14] and [3]). In terms of the rescaled variables and in cylindrical coordinates the King solutions are defined by a pressure function of the form

$$
u_{K}(x, \tau)=\xi(\tau)+\zeta(\tau) \cosh ((p-1) x), \quad x \in \mathbb{R}
$$

for some positive functions $\zeta(\tau), \xi(\tau)$ of rescaled time $\tau$. The function $u_{K}$ satisfies equation (1.16) and a direct calculation shows that this is equivalent to the system of ode's

$$
\left\{\begin{array}{l}
p \xi^{\prime}(\tau)=p(p-1) \zeta^{2}(\tau)+(p-1)\left(\xi^{2}(\tau)-\xi(\tau)\right) \\
p \zeta^{\prime}(\tau)=(p-1) \zeta(\tau)(-1+(p+1) \xi(\tau)) .
\end{array}\right.
$$

In the case where $\zeta(\tau) \equiv 0$, the above system is equivalent to the ode (1.25) which corresponds to space independent solutions of (1.16). The non-trivial solution $\xi(\tau)$ of this equation is given by (1.26) for a parameter $k \geq 0$ and geometrically corresponds to cylinders evolving by the Yamabe flow.

In all other cases, a simple analysis shows that

$$
\xi(\tau)=1+k e^{\frac{p-1}{p} \tau}+o\left(e^{\frac{p-1}{p} \tau}\right), \quad \zeta(\tau)=c e^{(p-1) \tau}+o\left(e^{(p-1) \tau}\right),
$$

for some parameters $k>0, c>0$.

Now let us relate the King solutions to the Barenblatt self-similar solutions (1.19) and our approximating supersolutions $w_{\lambda, \lambda^{\prime}, h, h^{\prime}, k}$ given by (1.30). Consider the approximating solutions defined by

$$
w_{K, h, k}(x, \tau)=v_{1}(x-\tau-h)+v_{1}(-x-\tau-h)+\xi_{k}(\tau)-2
$$

with $v_{1}$ and $\xi_{k}$ given by (1.19) and (1.26) respectively. It follows that

$$
w_{K, h, k}(x, \tau)=c e^{(p-1) \tau} \cosh ((p-1) x)+\xi_{k}(\tau)
$$

for a parameter $c$ depending on $h$. Using the asymptotics (1.27) it follows that $w_{K, h, k}(x, \tau)$ is the first order approximation of (2.1) as shown in (2.3). This shows that that our approximations $w_{\lambda, \lambda^{\prime}, h, h^{\prime}, k}$ given by (1.30) is a natural extension of the King solutions to the case of $\lambda>1$ where the traveling waves (solitons) $v_{\lambda}$ are not given in closed form. We will see in the next section that the exponentially small term $\lambda q e^{\frac{p-1}{p} \tau}$ which is subtracted from the traveling wave speed $\lambda$ of the traveling wave $v_{\lambda}$ in the definition (1.30), plays an essential role into making $w_{\lambda, \lambda^{\prime}, h, h^{\prime}, k}^{+}$to be a supersolution.

$$
\text { 3. The Supersolution } w_{\lambda, \lambda^{\prime}, h, h^{\prime}, k}^{+}
$$

In this section we will show that

$$
w_{\lambda, \lambda^{\prime}, h, h^{\prime}, k}^{+}:=v_{\lambda}\left(x-\lambda \tau\left(1-q e^{\frac{p-1}{p} \tau}\right)+h\right)+v_{\lambda}\left(-x-\lambda \tau\left(1-q e^{\frac{p-1}{p} \tau}\right)+h\right)+\xi_{k}(\tau)-2
$$

is a supersolution of equation (1.16) for every $\left(\lambda, \lambda^{\prime}, h, h^{\prime}, k\right) \in \mathbb{R}^{5}$ with $\lambda, \lambda^{\prime}>1, k \geq 0$ and $h, h^{\prime} \in \mathbb{R}$ if $q=q(p)>0$ is chosen appropriately. 
To simplify the notation, we will fix for the remainder of this section $\left(\lambda, \lambda^{\prime}, h, h^{\prime}, k\right) \in \mathbb{R}^{5}$ as above and we will simply denote by $w:=w_{\lambda, \lambda^{\prime}, h, h^{\prime}, k}^{+}$and

$$
w_{1}(x, \tau):=v_{\lambda}\left(x-\lambda \tau\left(1-q e^{\tau / 2}\right)+h\right), w_{2}(\tau):=\xi_{k}(\tau)-1, w_{3}(x, \tau):=v_{\lambda^{\prime}}\left(-x-\lambda^{\prime} \tau\left(1-q e^{\tau / 2}\right)+h^{\prime}\right) .
$$

Also, we will set

$$
z:=x-\lambda \tau\left(1-q e^{\frac{p-1}{p} \tau}\right)+h \quad \text { and } \quad \bar{z}:=-x-\lambda^{\prime} \tau\left(1-q e^{\frac{p-1}{p} \tau}\right)+h^{\prime} .
$$

We recall that $v_{\lambda}, v_{\lambda^{\prime}}$ satisfy (1.17) and $\xi_{k}$ satisfies (1.25). It follows that $w_{2}:=\xi_{k}-1$ satisfies the ode

$$
p\left(w_{2}\right)_{\tau}=(p-1)\left(w_{2}^{2}+w_{2}\right) .
$$

We then have by (3.1) that

$$
w:=w_{1}+w_{2}+w_{3}-1 .
$$

Define next the operator

$$
L(w):=w w_{x x}-\frac{p}{p-1} w_{x}^{2}+(p-1)\left(w^{2}-w\right)-p w_{\tau} .
$$

A direct computation shows that

$$
\begin{aligned}
L(w) & =\left(\sum_{i=1}^{3} w_{i}-1\right) \sum_{i=1}^{3}\left(w_{i}\right)_{x x}-\frac{p}{p-1}\left(\sum_{i=1}^{3}\left(w_{i}\right)_{x}\right)^{2}+(p-1)\left(\sum_{i=1}^{3} w_{i}-1\right)^{2} \\
& -(p-1)\left(\sum_{i=1}^{3} w_{i}-1\right)-p\left(\sum_{i=1}^{3}\left(w_{i}\right)_{\tau}\right) \\
& =\sum_{i, j=1}^{3} w_{i}\left(w_{j}\right)_{x x}-\sum_{i=1}^{3}\left(w_{i}\right)_{x x}-\frac{p}{p-1} \sum_{i, j=1}^{3}\left(w_{i}\right)_{x}\left(w_{j}\right)_{x}+(p-1) \sum_{i, j=1}^{3} w_{i} w_{j} \\
& -3(p-1) \sum_{i=1}^{3} w_{i}+2(p-1)-p\left(\sum_{i=1}^{3}\left(w_{i}\right)_{\tau}\right) .
\end{aligned}
$$

Since

$$
\sum_{i=1}^{3}\left(w_{i}\right)_{\tau}=\sum_{i=1,3}\left(w_{i}\right)_{\tau}+\left(w_{2}\right)_{\tau}=\left(w_{2}\right)_{\tau}-\left(\lambda v_{\lambda}^{\prime}+\lambda^{\prime} v_{\lambda^{\prime}}\right)+q \lambda\left(\tau e^{\frac{p-1}{p} \tau}\right)^{\prime} v_{\lambda}^{\prime}+q \lambda^{\prime}\left(\tau e^{\frac{p-1}{p} \tau}\right)^{\prime} v_{\lambda^{\prime}}^{\prime}
$$

and $\left(w_{1}\right)_{x}=v_{\lambda}^{\prime},\left(w_{3}\right)_{x}=-v_{\lambda^{\prime}}^{\prime}$ we have

$$
\sum_{i=1}^{3}\left(w_{i}\right)_{\tau}=\left(w_{2}\right)_{\tau}-\left(\lambda\left(w_{1}\right)_{x}-\lambda^{\prime}\left(w_{3}\right)_{x}\right)+q \lambda\left(\tau e^{\frac{p-1}{p} \tau}\right)^{\prime}\left(w_{1}\right)_{x}-q \lambda^{\prime}\left(\tau e^{\frac{p-1}{p} \tau}\right)^{\prime}\left(w_{3}\right)_{x} .
$$

Hence,

$$
\begin{aligned}
L(w)= & \sum_{i, j=1}^{3} w_{i}\left(w_{j}\right)_{x x}-\sum_{i=1}^{3}\left(w_{i}\right)_{x x}-\frac{p}{p-1} \sum_{i, j=1}^{3}\left(w_{i}\right)_{x}\left(w_{j}\right)_{x}+(p-1) \sum_{i, j=1}^{3} w_{i} w_{j} \\
& -3(p-1) \sum_{i=1}^{3} w_{i}+2(p-1)-p\left(w_{2}\right)_{\tau}+p\left(\lambda\left(w_{1}\right)_{x}-\lambda^{\prime}\left(w_{3}\right)_{x}\right) \\
& -p q \lambda\left(\tau e^{\frac{p-1}{p} \tau}\right)^{\prime}\left(w_{1}\right)_{x}+p q \lambda^{\prime}\left(\tau e^{\frac{p-1}{p} \tau}\right)^{\prime}\left(w_{3}\right)_{x} .
\end{aligned}
$$


Since both $v_{\lambda}, v_{\lambda^{\prime}}$ satisfy (1.17) and $w_{2}$ satisfies (3.3), the above yields

$$
\begin{aligned}
L(w) & =w_{1}\left(w_{3}\right)_{x x}+w_{3}\left(w_{1}\right)_{x x}+\left(w_{2}-1\right)\left(\left(w_{1}\right)_{x x}+\left(w_{3}\right)_{x x}\right)-\frac{2 p}{p-1}\left(w_{1}\right)_{x}\left(w_{3}\right)_{x} \\
& +2(p-1)\left(w_{1} w_{3}-1\right)-2(p-1) \sum_{i=1,3}\left(w_{i}-1\right)+2(p-1) w_{2}\left(w_{1}+w_{3}\right) \\
& -4(p-1) w_{2}-p q \lambda\left(\tau e^{\frac{p-1}{p} \tau}\right)^{\prime}\left(w_{1}\right)_{x}+p q \lambda^{\prime}\left(\tau e^{\frac{p-1}{p} \tau}\right)^{\prime}\left(w_{3}\right)_{x} .
\end{aligned}
$$

Finally,

$$
\begin{aligned}
L(w)= & \left(w_{3}\right)_{x x}\left(w_{1}+w_{2}-1\right)+\left(w_{1}\right)_{x x}\left(w_{3}+w_{2}-1\right)-\frac{2 p}{p-1}\left(w_{1}\right)_{x}\left(w_{3}\right)_{x} \\
& +2(p-1)\left(w_{1}-1\right)\left(w_{3}-1\right)+2(p-1) w_{2}\left(w_{1}+w_{3}-2\right) \\
& -p q \lambda\left(\tau e^{\frac{p-1}{p} \tau}\right)^{\prime}\left(w_{1}\right)_{x}+p q \lambda^{\prime}\left(\tau e^{\frac{p-1}{p} \tau}\right)^{\prime}\left(w_{3}\right)_{x} .
\end{aligned}
$$

Notice that $\left(w_{1}\right)_{x}=v_{\lambda}^{\prime} \geq 0$ and $\left(w_{2}\right)_{x}=-v_{\lambda^{\prime}}^{\prime} \leq 0$, which implies that the last two terms in (3.5) are negative. We will see in what follows that those two terms play a crucial role for making $w$ a supersolution. In fact, we will next use (3.5) to show that $w$ is a supersolution of (1.16).

Lemma 3.1. There exists a number $\tau_{0}<<0$ depending on $p$ and $\lambda, \lambda^{\prime}, h, h^{\prime}, k$ for which the function $w:=w_{\lambda, \lambda^{\prime}, h, h^{\prime}, k}^{+}$defined as in (3.4) is a supersolution of equation (1.16), that is $L w \leq 0$ on $\mathbb{R} \times\left(-\infty, \tau_{0}\right]$.

Proof. The monotonicity of $v_{\lambda}, v_{\lambda^{\prime}}$ implies that $w_{1}(x, \tau)$ is monotone decreasing in $x$ and $w_{2}(x, \tau)$ is monotone increasing in $x$. Hence, the asymptotic behavior in (1.18) implies that for each $\tau<<0$ there exists a unique $x(\tau) \in \mathbb{R}$ at which $w_{1}(x(\tau), \tau)=w_{2}(x(\tau), \tau)$. In addtion, it follows from (1.18) that as $\tau \rightarrow-\infty$, the point $x(\tau)$ satisfies the asymptotic behavior

$$
x(\tau)=\frac{\gamma_{\lambda}-\gamma_{\lambda^{\prime}}}{p} \tau+\frac{1}{\gamma_{\lambda}+\gamma_{\lambda^{\prime}}}\left(\ln \frac{C_{\lambda}}{C_{\lambda^{\prime}}}+h^{\prime} \gamma_{\lambda^{\prime}}-h \gamma_{\lambda}\right)+o(1) .
$$

and that at $x=x(\tau)$ we have

$$
w_{1}(x(\tau), \tau)=w_{2}(x(\tau), \tau)=1-C_{\lambda, \lambda^{\prime}, h, h^{\prime}, k} e^{d \tau}+o\left(e^{d \tau}\right)
$$

with

$$
d:=\frac{\gamma_{\lambda} \gamma_{\lambda^{\prime}}+(p-1)}{p} .
$$

and $C_{\lambda, \lambda^{\prime}, h, h^{\prime}, k}$ depending on $\lambda, \lambda^{\prime}, h, h^{\prime}, k$.

Trying to show that $L w \leq 0$, for all $x \in \mathbb{R}, \tau \leq \tau_{0}<<0$, we observe first that by symmetry it is sufficient to consider the region $x \leq x(\tau), \tau \leq \tau_{0}$. Recalling that $z:=x-\lambda \tau\left(1-q e^{\frac{p-1}{p} \tau}\right)+h$, we will next distinguish between the three different cases $z \leq-M,-M \leq z \leq M$ and $M \leq z \leq x(\tau)-\lambda \tau\left(1-q e^{\frac{p-1}{p} \tau}\right)+h$.

Case 1. $z \leq-M$.

This implies $x \leq-M+\lambda \tau\left(1-q e^{\frac{p-1}{p} \tau}\right)-h$ and hence $\bar{z} \geq M-\left(\lambda+\lambda^{\prime}\right) \tau\left(1-q e^{\frac{p-1}{p} \tau}\right)+h+h^{\prime}$. By (1.22) we have that in the considered region the following asymptotics (up to a constant, whether positive or negative) hold

$$
w_{1}(x, \tau) \sim e^{-(p-1) z}, \quad w_{2}(\tau)=k e^{\frac{p-1}{p} \tau}+o\left(e^{\frac{p-1}{p} \tau}\right), \quad w_{3}(x, \tau) \sim 1+C_{p} e^{-\gamma_{\lambda^{\prime}} \bar{z}} .
$$

As in [6] we can argue that up to a time independent constant (whether positive or negative), in the considered region we have

$$
w_{1} \sim\left(w_{1}\right)_{x}, \quad\left(w_{1}\right)_{x x} \sim\left(w_{1}\right)_{x}, \quad\left(w_{3}\right)_{x} \sim e^{-\gamma_{\lambda^{\prime}} \bar{z}}, \quad\left(w_{3}\right)_{x x} \sim e^{-\gamma_{\lambda^{\prime}} \bar{z}} .
$$


On the other hand, since $\bar{z} \geq M-\left(\lambda+\lambda^{\prime}\right) \tau\left(1-q e^{\frac{p-1}{p} \tau}\right)+h+h^{\prime}$, we have

$$
e^{-\gamma_{\lambda^{\prime}} \bar{z}} \leq C e^{\left(\lambda+\lambda^{\prime}\right) \gamma_{\lambda^{\prime}} \tau}=o\left(e^{\lambda^{\prime} \gamma_{\lambda^{\prime}} \tau}\right)=o\left(e^{\frac{p-1}{p} \tau}\right)
$$

where the last equality holds because $\lambda^{\prime} \gamma_{\lambda^{\prime}}>\frac{p-1}{p}$.

Lets now analyze terms in (3.5). We claim the prevailing term is $-(p-1) q \lambda \tau e^{\frac{p-1}{p} \tau}\left(w_{1}\right)_{x}<0$, and that the others can be absorbed in this one, at least when $\tau<<0$. It turns out the constants (whether positive or negative) for the other terms in (3.5) are not important and hence we will only emphasize the behavior. Using (3.9) and (3.10) we get

$$
\left(w_{3}\right)_{x x}\left(w_{1}+w_{2}-1\right) \sim\left(w_{3}\right)_{x x} w_{1} \sim o\left(e^{\frac{p-1}{p} \tau}\right)\left(w_{1}\right)_{x}
$$

and

$$
\left(w_{1}\right)_{x x}\left(w_{3}+w_{2}-1\right) \sim\left(w_{1}\right)_{x}\left(w_{3}-1\right)+\left(w_{1}\right)_{x} w_{2}=O\left(e^{\frac{p-1}{p} \tau}\right)\left(w_{1}\right)_{x}
$$

Similarly, we get

$$
-\frac{2 p}{p-1}\left(w_{1}\right)_{x}\left(w_{3}\right)_{x}+2(p-1)\left(w_{1}-1\right)\left(w_{3}-1\right)+2(p-1) w_{2}\left(w_{1}+w_{3}-2\right)=O\left(e^{\frac{p-1}{p} \tau}\right)\left(w_{1}\right)_{x} .
$$

Combining the above finally yields

$$
L(w) \leq-(p-1) q \lambda \tau e^{\frac{p-1}{p} \tau}\left(w_{1}\right)_{x}+o\left(\tau e^{\frac{p-1}{p} \tau}\left(w_{1}\right)_{x}\right)<0
$$

for $\tau<<0$.

Case 2. $-M \leq z \leq M$.

This is equivalent to $-M+\lambda \tau\left(1-q e^{\frac{p-1}{p} \tau}\right)-h \leq x \leq M+\lambda \tau\left(1-q e^{\frac{p-1}{p} \tau}\right)-h$ which implies that $\bar{z} \geq-M-\left(\lambda+\lambda^{\prime}\right) \tau\left(1-q e^{\frac{p-1}{p} \tau}\right)+h+h^{\prime}$. By (1.22), in the considered region we have

$$
a \leq w_{1}(x, \tau) \leq b, \quad w_{2}(\tau)=k e^{\frac{p-1}{p} \tau}+o\left(e^{\frac{p-1}{p} \tau}\right), \quad w_{3}(x, \tau) \sim 1+C_{p} e^{-\gamma_{\lambda^{\prime}} \bar{z}}, \quad e^{-\gamma_{\lambda^{\prime}} \bar{z}}=o\left(e^{\frac{p-1}{p} \tau}\right)
$$

for some constants $a, b>0$. As before, we have

$$
\left(w_{3}\right)_{x} \sim e^{-\gamma_{\lambda^{\prime}} \bar{z}}, \quad\left(w_{3}\right)_{x x} \sim e^{-\gamma_{\lambda^{\prime}} \bar{z}}
$$

up to a constant (positive or negative). Analyzing terms in (3.5) and using (3.10)

$$
a_{1} \leq\left(w_{1}\right)_{x} \leq b_{1}, \quad\left|\left(w_{1}\right)_{x x}\right| \leq C, \quad\left(w_{3}\right)_{x}=o\left(e^{\frac{p-1}{p} \tau}\right), \quad\left(w_{3}\right)_{x x}=o\left(e^{\frac{p-1}{p} \tau}\right)
$$

we find

$$
\left(w_{3}\right)_{x x}\left(w_{1}+w_{2}-1\right)=o\left(e^{\frac{p-1}{p} \tau}\right), \quad\left(w_{1}\right)_{x x}\left(w_{3}+w_{2}-1\right)=O\left(e^{\frac{p-1}{p} \tau}\right) .
$$

Similarly, we get

$$
-\frac{2 p}{p-1}\left(w_{1}\right)_{x}\left(w_{3}\right)_{x}+2(p-1)\left(w_{1}-1\right)\left(w_{3}-1\right)+2(p-1) w_{2}\left(w_{1}+w_{3}-2\right)=O\left(e^{\frac{p-1}{p} \tau}\right) .
$$

Hence, for $-M \leq z \leq M$, we conclude

$$
L(w) \leq-(p-1) q \lambda \tau e^{\frac{p-1}{p} \tau}\left(w_{1}\right)_{x}+o\left(\tau e^{\frac{p-1}{p} \tau}\right) \leq-(p-1) q \lambda a_{1} \tau e^{\frac{p-1}{p} \tau}+o\left(\tau e^{\frac{p-1}{p} \tau}\right)<0
$$

for $\tau<<0$.

Case 3. $M \leq z \leq x(\tau)-\lambda \tau\left(1-q e^{\frac{p-1}{p} \tau}\right)+h$. 
Using (3.6), in this case we have

$$
M \leq z \leq x(\tau)-\lambda \tau\left(1-q e^{\frac{p-1}{p} \tau}\right)+h=-\frac{\left(\gamma_{\lambda} \gamma_{\lambda^{\prime}}+(p-1)\right)}{p \gamma_{\lambda}} \tau+C\left(\lambda, \lambda^{\prime}, h, h^{\prime}, p\right)+o(1) .
$$

Also, $M+\lambda \tau\left(1-q e^{\frac{p-1}{p} \tau}\right)-h \leq x \leq x(\tau)$ implies that

$$
\bar{z} \geq-x(\tau)-\lambda^{\prime} \tau\left(1-q e^{\frac{p-1}{p} \tau}\right)+h^{\prime}=-\frac{\gamma_{\lambda}\left(\lambda+\lambda^{\prime}\right)}{\gamma_{\lambda}+\gamma_{\lambda^{\prime}}} \tau+C\left(\lambda, \lambda^{\prime}, h, h^{\prime}, p\right)+o(1) .
$$

It follows that in the considered region both $z>>1$ and $\bar{z}>>1$, hence

$$
w_{1}=1+C_{p} e^{-\gamma_{\lambda} z}, \quad\left(w_{1}\right)_{x} \sim-C_{p} e^{-\gamma_{\lambda} z}, \quad\left(w_{1}\right)_{x x} \sim C_{p} e^{-\gamma_{\lambda} z}
$$

and

$$
w_{3}=1+C_{p}^{\prime} e^{-\gamma_{\lambda^{\prime}} \bar{z}}, \quad\left(w_{3}\right)_{x} \sim C_{p}^{\prime} e^{-\gamma_{\lambda^{\prime}} \bar{z}}, \quad\left(w_{3}\right)_{x x} \sim C_{p}^{\prime} e^{-\gamma_{\lambda^{\prime}} \bar{z}}
$$

up to a positive constant, with $C_{p}>0, C_{p}^{\prime}>0$. Analyzing terms in (3.5), we have

$$
\begin{aligned}
\left(w_{3}\right)_{x x}\left(w_{1}+w_{2}-1\right) & \sim C_{1} e^{-\gamma_{\lambda^{\prime}} \bar{z}} e^{\frac{p-1}{p} \tau}+C_{2} e^{-\gamma_{\lambda^{\prime}} \bar{z}} e^{-\gamma_{\lambda} z} \\
& \sim\left(w_{1}\right)_{x}\left(C_{1} e^{\frac{p-1}{p} \tau} e^{-\gamma_{\lambda^{\prime}} \bar{z}+\gamma_{\lambda} z}+C_{2} e^{-\gamma_{\lambda^{\prime}} \bar{z}}\right)
\end{aligned}
$$

with $C_{1}, C_{2}>0$. Note that

$$
e^{-\gamma_{\lambda^{\prime}} \bar{z}} \leq e^{\frac{\gamma_{\lambda} \gamma_{\lambda^{\prime}}\left(\lambda+\lambda^{\prime}\right) \tau}{\gamma_{\lambda}+\gamma_{\lambda^{\prime}}}} \leq C e^{\frac{p-1}{p} \tau}
$$

since $\lambda \gamma_{\lambda} \geq \frac{p-1}{p}$. Furthermore,

$$
e^{-\gamma_{\lambda^{\prime}} \bar{z}+\gamma_{\lambda} z} \leq e^{\gamma_{\lambda}(x(\tau)-\lambda \tau)-\gamma_{\lambda^{\prime}}\left(-x(\tau)-\lambda^{\prime} \tau\right)} \leq C
$$

due to a definition of $x(\tau)$. Overall we have,

$$
\left(w_{3}\right)_{x x}\left(w_{1}+w_{2}-1\right) \leq C e^{\frac{p-1}{p} \tau}\left(w_{1}\right)_{x}=o\left(\left(\tau e^{\frac{p-1}{p} \tau}\right)\left(w_{1}\right)_{x}\right) .
$$

Similarly we analyze other terms in (3.5) of the same form in the considered region and we also use that $p q \lambda^{\prime}\left(\tau e^{\frac{p-1}{p} \tau}\right)^{\prime}\left(w_{3}\right)_{x} \leq 0$, to conclude that the dominating term is $-p q \lambda\left(\tau e^{\frac{p-1}{p} \tau}\right)^{\prime}\left(w_{1}\right)_{x}<0$, hence

$$
L(w) \leq-(p-1) q \lambda \tau e^{\frac{p-1}{p} \tau}\left(w_{1}\right)_{x}+o\left(\tau e^{\frac{p-1}{p} \tau}\right)<0
$$

for $\tau<<0$.

Finally, above analysis in cases 1-3 yields that $L(w) \leq 0$, meaning that $w$ is a supersolution as stated in the Lemma.

\section{The existence of a five Parameter family of AnCient solutions}

In this section we show the the existence of a five parameter ancient solution $u_{\lambda, \lambda^{\prime}, h, h^{\prime}, k}$ of (1.16), as stated in the next theorem.

Theorem 4.1. For any $\left(\lambda, \lambda^{\prime}, h, h^{\prime}, k\right) \in \mathbb{R}^{5}$ such that $\lambda, \lambda^{\prime}>1, k \geq 0$, there exists an ancient solution $u_{\lambda, \lambda^{\prime}, h, h^{\prime}, k}$ to (1.16) defined on $\mathbb{R} \times(-\infty, T)$, for some $T=T_{\lambda, \lambda^{\prime}, h, h^{\prime}, k} \in(-\infty, \infty]$. Moreover,

$$
w_{\lambda, \lambda^{\prime}, h, h^{\prime}, k}^{-}(x, \tau) \leq u_{\lambda, \lambda^{\prime}, h, h^{\prime}, k}(x, \tau) \leq w_{\lambda, \lambda^{\prime}, h, h^{\prime}, k}^{+}(x, \tau)
$$

where $w_{\lambda, \lambda^{\prime}, h, h^{\prime}, k}^{-}$and $w_{\lambda, \lambda^{\prime}, h, h^{\prime}, k}^{+}$are defined by (1.29) and (1.30), respectively. 
Proof. We have seen in Lemma 3.1 that $w_{\lambda, \lambda^{\prime}, h, h^{\prime}, k}^{+}$is a supersolution of (1.16) for $\tau \leq \tau_{0}$ with $\tau_{0}<<$. For any $m \in \mathbb{N}$ with $-m<\tau_{0}$, let $u_{m}$ denote the solution of the initial value problem

$$
\begin{cases}p u_{\tau}=u u_{x x}-\frac{p}{p-1} u_{x}^{2}+(p-1)\left(u^{2}-u\right) & x \in \mathbb{R}, \tau>-m \\ u(\cdot,-m)=w_{\lambda, \lambda^{\prime}, h, h^{\prime}, k}^{+}(\cdot,-m) & x \in \mathbb{R}\end{cases}
$$

with exponent $p=\frac{n+2}{n-2}>1$. By Lemma 3.1 and the comparison principle we immediately get that

$$
u_{m}(x, \tau) \leq w_{\lambda, \lambda^{\prime}, h, h^{\prime}, k}^{+}(x, \tau), \quad-m \leq \tau \leq \tau_{0} .
$$

On the other hand, since $v_{\lambda}(x-\lambda \tau+h), v_{\lambda^{\prime}}\left(-x-\lambda^{\prime} \tau+h^{\prime}\right)$ and $\xi_{k}(\tau)$ are all solutions to (1.16), it follows that $w_{\lambda, \lambda^{\prime}, h, h^{\prime}, k}^{-}(x, \tau):=\min \left(v_{\lambda}(x-\lambda \tau+h), \xi_{k}(\tau), v_{\lambda^{\prime}}\left(-x-\lambda^{\prime} \tau+h^{\prime}\right)\right)$ is a subsolution of (1.16). Furthermore, we claim that

$$
w_{\lambda, \lambda^{\prime}, h, h^{\prime}, k}^{-}(x, \tau) \leq u_{m}(x, \tau), \quad \text { for } \tau<0
$$

which readily gives

$$
w_{\lambda, \lambda^{\prime}, h, h^{\prime}, k}^{-}(x,-m) \leq u_{m}(x,-m) .
$$

To see (4.2), we recall that $v_{\lambda}$ is monotone decreasing. Also that since all $v_{\lambda}, v_{\lambda^{\prime}}$ and $\xi_{k}$ are larger than equal to one, we have

$$
w_{\lambda, \lambda^{\prime}, h, h^{\prime}, k}^{+} \geq \max \left(v_{\lambda}\left(x-\lambda \tau\left(1-q e^{\frac{p-1}{p} \tau}\right)+h\right), \xi_{k}(\tau), v_{\lambda}\left(-x-\lambda \tau\left(1-q e^{\frac{p-1}{p} \tau}\right)+h\right)\right) .
$$

Hence, on the regions where $w_{\lambda, \lambda^{\prime}, h, h^{\prime}, k}^{-}(x, \tau)=v_{\lambda}(x-\lambda \tau+h)$ and $w_{\lambda, \lambda^{\prime}, h, h^{\prime}, k}^{-}(x, \tau)=v_{\lambda^{\prime}}\left(-x-\lambda^{\prime} \tau+h^{\prime}\right)$, respectively, we have

$$
w_{\lambda, \lambda^{\prime}, h, h^{\prime}, k}^{-}(x, \tau)=v_{\lambda}(x-\lambda \tau+h) \leq v_{\lambda}\left(x-\lambda \tau\left(1-q e^{\frac{p-1}{p} \tau}\right)\right) \leq w_{\lambda, \lambda^{\prime}, h, h^{\prime}, k}^{+}(x, \tau)
$$

and

$$
w_{\lambda, \lambda^{\prime}, h, h^{\prime}, k}^{-}(x, \tau)=v_{\lambda^{\prime}}\left(-x-\lambda^{\prime} \tau+h^{\prime}\right) \leq v_{\lambda^{\prime}}\left(-x-\lambda^{\prime} \tau\left(1-q e^{\frac{p-1}{p} \tau}\right)+h^{\prime}\right) \leq w_{\lambda, \lambda^{\prime}, h, h^{\prime}, k}^{+}(x, \tau) .
$$

Finally, on the region where $w_{\lambda, \lambda^{\prime}, h, h^{\prime}, k}^{-}(x, \tau)=\xi_{k}(\tau)$ we immediately have

$$
w_{\lambda, \lambda^{\prime}, h, h^{\prime}, k}^{-}(x, \tau)=\xi_{k}(\tau) \leq w_{\lambda, \lambda^{\prime}, h, h^{\prime}, k}^{+}(x, \tau) .
$$

It follows that (4.2) holds in all regions, thus $w_{\lambda, \lambda^{\prime}, h, h^{\prime}, k}^{-}(x,-m)=\xi_{k}(\tau) \leq u_{m}(x,-m)$ and by the comparison principle we $w_{\lambda, \lambda^{\prime}, h, h^{\prime}, k}^{-}(x, \tau) \leq u_{m}(x, \tau)$, for all $\tau \geq-m$. We conclude from the discussion above that

$$
w_{\lambda, \lambda^{\prime}, h, h^{\prime}, k}^{-}(x, \tau) \leq u_{m}(x, \tau) \leq w_{\lambda, \lambda^{\prime}, h, h^{\prime}, k}^{+}(x, \tau), \quad-m \leq \tau \leq \tau_{0} .
$$

It follows from standard arguments in quasilinear parabolic equations that the sequence of solutions $\left\{u_{m}\right\}_{\left\{m<\tau_{0}\right\}}$ is equicontinuous on compact subsets of $\mathbb{R} \times\left(-\infty, \tau_{0}\right)$, hence passing to a subsequence $u_{m_{k}}$ it converges uniformly on compact subsets of $\mathbb{R} \times\left(-\infty, \tau_{0}\right)$ to a smooth ancient solution $u_{\lambda, \lambda^{\prime}, h, h^{\prime}, k}$ of (1.16). In addition, $u$ satisfies

$$
w_{\lambda, \lambda^{\prime}, h, h^{\prime}, k}^{-}(x, \tau) \leq u_{\lambda, \lambda^{\prime}, h, h^{\prime}, k}(x, \tau) \leq w_{\lambda, \lambda^{\prime}, h, h^{\prime}, k}^{+}(x, \tau), \quad-m \leq \tau \leq \tau_{0} .
$$

We will next observe that $u_{\lambda, \lambda^{\prime}, h, h^{\prime}, k}$ defines a smooth ancient solution of the rescaled Yamabe flow on $S^{n} \times\left(-\infty, T_{\lambda, \lambda^{\prime}, h, h^{\prime}, k}\right)$, for some maximal time $T_{\lambda, \lambda^{\prime}, h, h^{\prime}, k} \in(-\infty,+\infty]$ at which $u_{\lambda, \lambda^{\prime}, h, h^{\prime}, k}<+\infty$. 
Corollary 4.1. Set $\varphi_{\lambda, \lambda^{\prime}, h, h^{\prime}, k}:=u_{\lambda, \lambda^{\prime}, h, h^{\prime}, k}^{-\frac{1}{p-1}}$, where $u_{\lambda, \lambda^{\prime}, h, h^{\prime}, k}$ is an ancient solution of (1.16) given by Theorem 4.1. Then, $\varphi_{\lambda, \lambda^{\prime}, h, h^{\prime}, k}$ defines a solution of (1.8) which is equivalent to having a smooth solution of the rescaled Yamabe flow $g_{t}=-(R-1) g$ on $S^{n} \times\left(-\infty, T_{\lambda, \lambda^{\prime}, h, h^{\prime}, k}\right)$.

Proof. It is clear that $\varphi_{\lambda, \lambda^{\prime}, h, h^{\prime}, k}:=u_{\lambda, \lambda^{\prime}, h, h^{\prime}, k}^{-\frac{1}{p-1}}$ is a smooth ancient solution of (1.8) which in addition satisfies

$$
w_{\lambda, \lambda^{\prime}, h, h^{\prime}, k}^{+}(x, \tau)^{-\frac{1}{p-1}} \leq \varphi_{\lambda, \lambda^{\prime}, h, h^{\prime}, k}(x, \tau) \leq w_{\lambda, \lambda^{\prime}, h, h^{\prime}, k}^{-}(x, \tau)^{-\frac{1}{p-1}}, \quad x \in \mathbb{R},-m \leq \tau \leq \tau_{0} .
$$

Notice next that $w_{\lambda, \lambda^{\prime}, h, h^{\prime}, k}^{-}(\cdot, \tau)^{-\frac{1}{p-1}}$ and $w_{\lambda, \lambda^{\prime}, h, h^{\prime}, k}^{+}(\cdot, \tau)^{-\frac{1}{p-1}}$ define positive smooth metrics, when lifted on $S^{n}$. Thus, (4.4) implies that $\varphi_{\lambda, \lambda^{\prime}, h, h^{\prime}, k}(x, \tau)$, when lifted on $S^{n}$, also defines a smooth metric which equivalent (up to dilation) to a solution of the rescaled Yamabe flow $g_{t}=-(R-1) g$. Now standard results imply that there exists a maximal time $T_{\lambda, \lambda^{\prime}, h, h^{\prime}, k} \in(-\infty,+\infty]$ up to which this solution is defined, which means that $\varphi_{\lambda, \lambda^{\prime}, h, h^{\prime}, k}(x, t)>0$ for all $t<T_{\lambda, \lambda^{\prime}, h, h^{\prime}, k}$.

\section{Geometric properties of solutions}

In this last section we will derive the geometric properties of the ancient solution of the equation (1.8) as constructed in Theorem 4.1. We have observed in Corollary 4.1 that the one parameter family of metrics $g_{\lambda, \lambda^{\prime}, h, h^{\prime}, k}(\tau):=\varphi_{\lambda, \lambda^{\prime}, h, h^{\prime}, k}^{\frac{4}{n-2}}(\cdot, \tau) g_{c y l}$ can be lifted to a smooth one parameter family of metrics on $S^{n} \times\left(-\infty, T_{\lambda, \lambda^{\prime}, h, h^{\prime}, k}\right)$ which defines (up to dilation by constants) an ancient rotationally symmetric solution of the rescaled Yamabe flow on $S^{n}$, equation

$$
\frac{\partial}{\partial \tau} g=-(R-1) g
$$

We next prove the following result concerning the behavior of the Riemannian curvature of the metric $g_{\lambda, \lambda^{\prime}, h, h^{\prime}, k}(\tau)$ near $\tau=-\infty$.

Theorem 5.1. The solution $g_{\lambda, \lambda^{\prime}, h, h^{\prime}, k}(\tau):=\varphi_{\lambda, \lambda^{\prime}, h, h^{\prime}, k}^{\frac{4}{n-2}}(\cdot, \tau) g_{\text {cyl }}$ defines a type I ancient solution to the Yamabe flow in the sense that, for any $\tau_{0}<T_{\lambda, \lambda^{\prime}, h, h^{\prime}, k}$, the norm of its curvature operator satisfies the uniform bound

$$
\left\|\operatorname{Rm}\left(g_{\lambda, \lambda^{\prime}, h, h^{\prime}, k}\right)\right\| \leq C, \quad \text { for all } \tau \leq \tau_{0} .
$$

Remark 5.1. The statement of Theorem 5.1 exactly means that the unrescaled flow (1.1), whose scaling by $|t|$ yields to the equation (5.1), is a type I ancient solution according to the Definition 1.1.

Proof of Theorem 5.1. It is sufficient to prove the bound (5.2) for $\tau_{0}<<0$. Since our metric $g_{\lambda, \lambda^{\prime}, h, h^{\prime}, k}$ is conformally flat, the norm $\|\mathrm{Rm}\|$ of its curvature operator can be expressed in terms of powers (positive or negative) of the conformal factor and its first and second order derivatives. On the other hand, since the conformal factor (in any parametrization) satisfies a quasilinear parabolic equation, it follows by standard parabolic estimates, that uniform upper and lower bounds away from zero on the conformal factor imply uniform bounds on all its derivatives and therefore the desired uniform bound on $\|\mathrm{Rm}\|$. By Corollary 4.1, we have $g_{\lambda, \lambda^{\prime}, h, h^{\prime}, k}=\varphi^{\frac{4}{n-2}} g_{c y l}$, where $\varphi$ is a solution of (1.8) and satisfies $0<\varphi<1$, for all $\tau<T_{\lambda, \lambda^{\prime}, h, h^{\prime}, k}$. Notice that in order to simplify the notation we have dropped the index $\lambda, \lambda^{\prime}, h, h^{\prime}, k$ from $\varphi_{\lambda, \lambda^{\prime}, h, h^{\prime}, k}$ and simply denote it by $\varphi$. It follows by the discussion above that in the region where $1 / 2<\varphi<1$ the bound $\|\mathrm{Rm}\| \leq C$ readily holds. However, since $\varphi(x, \tau) \rightarrow 0$, as $x \rightarrow \pm \infty$, in the region where $0<\varphi<1 / 2$ we 
will obtain the desired bound by lifting the metric $g_{\lambda, \lambda^{\prime}, h, h^{\prime}, k}$ on $\mathbb{R}^{n}$ and showing that in the considered region if $g_{\lambda, \lambda^{\prime}, h, h^{\prime}, k}=\hat{\varphi}^{\frac{4}{n-2}} g_{\mathbb{R}^{n}}$, then $c \leq \hat{\varphi} \leq C$ for some positive constants $c, C$ which are uniform in $\tau$.

Observe that is sufficient to establish the uniform bound on $\|\mathrm{Rm}\|$ for $x \leq x(\tau)$, where $x(\tau)$ is given by (3.6), since in the remaining region the estimate could be proved in a similar manner. We go from cylindrical to polar coordinates via the following coordinate change,

$$
\Phi(z, \tau)=\hat{\varphi}(y, \tau)|y|^{\frac{2}{p-1}}, \quad r=|y|=e^{\frac{p-1}{2} z}
$$

where $z:=x-\lambda \tau+h$ and $\Phi(z, \tau):=\varphi(z+\lambda \tau-h)$. It follows by a direct calculation that $\hat{\varphi}(y, \tau)$ satisfies the equation

$$
\left(\hat{\varphi}^{p}\right)_{\tau}=\alpha \Delta \hat{\varphi}+\beta r \hat{\varphi}_{r}+\gamma \hat{\varphi}
$$

for some constants $\alpha>0$ and $\beta, \gamma$. For any $M>0$, the compact region $|y| \leq 2 M$ corresponds in cylindrical coordinates to the region

$$
x \leq \lambda \tau-h+\frac{2}{p-1} \log 2 M=: \bar{x}_{M}(\tau) .
$$

Note that $\lambda p>\gamma_{\lambda}>\gamma_{\lambda}-\gamma_{\lambda^{\prime}}$, implying that $\bar{x}_{M}(\tau)<<x(\tau)$ holds, for $\tau<<0$.

Estimate (4.4) is crucial in proving our bound. Let us look first at the region $x \leq \bar{x}_{M}(\tau)$, which in polar coordinates corresponds to the region $|y| \leq M$. It follows from (4.4) that in this region we have

$$
\left(1-o_{M}(1)\right) \psi_{\lambda} \leq \varphi_{\lambda, \lambda^{\prime}, h, h^{\prime}, k} \leq \psi_{\lambda}
$$

for $\tau \leq \tau_{0}$ with $\tau_{0}<<0$, where we recall that $\psi_{\lambda}$ is the traveling wave solution of (1.10) which satisfies (1.12). In polar coordinates the above abound corresponds to

$$
c_{M} \leq \hat{\varphi} \leq C, \quad \text { for }|y| \leq M, \quad \tau \leq \tau_{0} .
$$

Having (5.5), equation (5.4) is parabolic equation for $(y, \tau) \in B(0,2 M) \times\left(-\infty, \tau_{0}\right)$, so standard parabolic estimates applied to equation (5.4) imply that we have uniform bounds on all the derivatives of $\hat{\varphi}$ in the region $B\left(0, \frac{3 M}{2}\right) \times\left(-\infty, 2 \tau_{0}\right)$. Since $\hat{\varphi}^{\frac{4}{n-2}}$ is the conformal factor of our metric $g_{\lambda, \lambda^{\prime}, h, h^{\prime}, k}$ in polar coordinates, by the previous discussion we have

$$
\|\operatorname{Rm}(y, \tau)\| \leq C, \quad|y| \leq M \quad \tau \leq 2 \tau_{0}
$$

for a uniform constant $C$. Equivalently we have a uniform curvature bound, in cylindrical coordinates, for all $x \leq \bar{x}_{M}(\tau)$. Observe that this estimate implies that the is curvature uniformly bounded in the tip region of our ancient solution.

Now fix $M>0$ and let us focus on the region $\bar{x}_{M}(\tau) \leq x \leq x(\tau)$ where our solution that turns out to have the asymptotics of a cylindrical metric at $\tau \rightarrow-\infty$. More precisely, we have $g_{\lambda, \lambda^{\prime}, h, h^{\prime}, k}=\varphi^{\frac{4}{n-2}} g_{c y l}$, where $\varphi$ is a solution of (1.8) and by (4.4) this region we may choose $\tau_{0}<<0$ such that

$$
1 / 2 \leq \varphi(x, \tau) \leq 1, \quad \text { for } \tau \leq \tau_{0} .
$$

The equation satisfied by $\varphi$ is therefore uniformly parabolic and hence we have uniform estimates on the derivatives of $\varphi$ in the inner region. As a result we have a uniform bound on $\|\mathrm{Rm}\|$ for all $\tau \leq \tau_{0}$ in that region as well. This concludes the proof of our bound.

\section{Acknowledgements}

P. Daskalopoulos has been partially supported by NSF grant DMS-1266172.

M. del Pino has been supported by grants Fondecyt 1150066 Fondo Basal CMM 
and Millenium Nucleus CAPDE NC130017.

N. Sesum has been partially supported by NSF grant DMS-1056387.

\section{REFERENCES}

[1] Brendle, S., Convergence of the Yamabe flow for arbitrary initial energy, J. Differential Geom. 69 (2005), $217-278$.

[2] Brendle, S., Convergence of the Yamabe flow in dimension 6 and higher, Invent. Math. 170 (2007), 541-576.

[3] Brendle, S., An analogue of the King-Rosenau solution for the Yamabe flow, personal communication.

[4] Cao, H-D., Sun, X., Zhang, Y., On the structure of gradient Yamabe solitons, Math. Res. Lett. 19 (2012), $767-774$.

[5] Chow, B., The Yamabe flow on locally conformally flat manifolds with positive Ricci curvature, Comm. Pure Appl. Math. 65 (1992), 1003-1014.

[6] Daskalopoulos, P., del Pino, M., King, J.R, Sesum, N., Type I ancient compact solutions to the Yamabe flow, to appear in Nonlinear Analysis: Theory, Methods \& Applications; arXiv:1509.08803.

[7] Daskalopoulos, P., del Pino, M., and Sesum N. Type II ancient compact solutions to the Yamabe flow, to appear in J. Reine Angew. Math., arXiv:1209.5479.

[8] Daskalopoulos, P., Hamilton, R. and Sesum, N., Classification of ancient solutions to the Ricci flow on surfaces, J. Differential Geom. 91 (2012), 171-214.

[9] Daskalopoulos, P., King, J.R. and Sesum, N., Extinction profile of complete non-compact solutions to the Yamabe flow, arXiv:1306.0859.

[10] Daskalopoulos, P., Sesum, N., Classification of conformally flat Yamabe solitons; Adv. Math. 240 (2013), $346-369$.

[11] del Pino, M.; Sáez, M., On the extinction profile for solutions of $u_{t}=\Delta u^{(N-2) /(N+2)}$. Indiana Univ. Math. J. 50 (2001), 611-628.

[12] Hamel, F.; Nadirashvili, N. Entire solutions of the KPP equation, Comm. Pure Appl. Math. 52 (1999), $1255-1276$.

[13] R. S. Hamilton, Lectures on geometric flows, 1989, unpublished.

[14] King, J.R., Exact polynomial solutions to some nonlinear diffusion equations, Physica. D 64 (1993), 39-65.

[15] King, J.R., Asymptotic results for nonlinear diffusion, European J. Appl. Math. 5 (1994), 359-390.

[16] Rosenau, P., Fast and superfast diffusion processes, Phys. Rev. Lett. 74 (1995), 1056-1059.

[17] Schoen, R., Conformal deformation of a Riemannian metric to constant scalar curvature, J. Differential Geom. 20 (1984) 479-495.

[18] Schwetlick, H.; Struwe, M. Convergence of the Yamabe flow for "large" energies, J. Reine Angew. Math. 562 (2003), 59-100.

[19] Vazquez, J.L., Asymptotic behaviour for the porous medium equation posed in the whole space, J.Evol.Equ. 3(2003), $67-118$.

[20] Vázquez, J. L., Smoothing and decay estimates for nonlinear diffusion equations, Oxford Lecture Series in Mathematics and its Applications 33 Oxford University Press, Oxford, 2006.

[21] Ye, R. Global existence and convergence of Yamabe flow, J. Differential Geom. 39 (1994), 35-50.

P. Daskalopoulos: Department of Mathematics, Columbia University, 2990 Broadway, New York, Ny 10027, USA.

E-mail address: pdaskalo@math.columbia.edu

M. del Pino: Departamento de Ingeniería Matemática and CMM, Universidad de Chile, Casilla 170 Correo 3, Santiago, Chile.

E-mail address: delpino@dim.uchile.cl

J. King: School of Mathematical Sciences, The University of Nottingham, University Park, Nottingham, NG7 2RD

E-mail address: john.king@nottingham.ac.uk

N. Sesum: Department of Mathematics, Rutgers University, 110 Frelinghuysen road, Piscataway, NJ 08854, USA.

E-mail address: natasas@math.rutgers.edu 